\title{
Quiste pericárdico gigante asintomático
}

\author{
Asymptomatic giant pericardial cyst
}

Héctor A. Carmona-Ruiz', Óscar Orihuela-Rodríguez ${ }^{2 *}$ y lan Morales-Gudiño

${ }^{1}$ Servicio de Cardiología, Hospital General de Zona y Medicina Familiar No. 1 Dr. Alfonso Mejía Schroeder, Instituto Mexicano del Seguro Social (IMSS), Pachuca de Soto, Hidalgo; '2Servicio de Cardiología, Unidad Médica de Alta Especialidad, Hospital de Especialidades Dr. Bernardo Sepúlveda Gutiérrez, Centro Médico Nacional Siglo XXI, IMSS, Ciudad de México. México

\section{Resumen}

Antecedentes: Los quistes pericárdicos son masas poco comunes; ocupan el tercer lugar entre las masas mediastinales quísticas. La mayoría son asintomáticos, pero se pueden asociar a complicaciones serias como tamponade cardiaco, obstrucción bronquial e incluso muerte súbita. Caso clínico: Mujer asintomática con cardiomegalia en la tele de tórax a quien se diagnostica de forma incidental, mediante ecocardiograma, un quiste pericárdico gigante, que se corroboró por resonancia magnética. Conclusiones: Los quistes pericárdicos pueden ser hallazgos incidentales en la radiografía de tórax. Tanto la tomografía computarizada como la resonancia magnética son estudios útiles para confirmar el diagnóstico.

Palabras clave: Quiste pericárdico. Ecocardiograma transtorácico. Resonancia magnética.

\begin{abstract}
Background: Pericardial cysts are uncommon masses and are the third most common cystic mass of the mediastinum. The majority are asymptomatic, however, they can be associated with serious complications such as cardiac tamponade, bronchial obstruction, or even sudden death. Case report: An asymptomatic female patient, who was referred due to a chest radiograph showing cardiomegaly. The transthoracic echocardiogram showed an image consistent with a pericardial cyst, the diagnosis was confirmed with a magnetic resonance imaging. Conclusions: Pericardial cysts may appear as an incidental finding in the chest radiograph, either computed tomography scan or magnetic resonance imaging are useful to confirm the diagnosis.
\end{abstract}

Key words: Pericardial cyst. Transthoracic echocardiography. Magnetic resonance imaging.

\section{Introducción}

Los quistes pericárdicos son masas poco comunes; ocupan el tercer lugar entre las masas mediastinales quísticas más comunes. La mayoría son asintomáticos y con un curso clínico benigno, pero en ocasiones pueden producir síntomas como disnea, dolor torácico o tos crónica. También se pueden asociar a complicaciones serias, como tamponade cardiaco, obstrucción bronquial e incluso muerte súbita ${ }^{1,2}$.

Los quistes pericárdicos gigantes son raros, con solo 12 casos reportados de 2000 a 2014. La mayoría son congénitos, aunque pueden presentarse desp ués de cirugías torácicas, pericarditis o trauma. La localización más frecuente es el ángulo cardiofrénico derecho.

\section{Correspondencia:}

*Óscar Orihuela-Rodríguez

Avda. Cuauhtémoc 330 
Se presenta el caso de una paciente con un quiste pericárdico gigante izquierdo asintomático ${ }^{1,2}$.

\section{Caso clínico}

Mujer de 56 años con antecedentes de hipotiroidismo y espondilitis anquilosante que fue referida al servicio de cardiología debido a que en la tele de tórax se observó cardiomegalia (Fig. 1). La paciente se encontraba asintomática y la exploración física fue normal.

Se le realizó, como parte del protocolo de estudio por cardiomegalia, un ecocardiograma transtorácico en el cual se observó una imagen hipoecoica sugestiva de quiste pericárdico de $8.7 \times 3.4 \mathrm{~cm}$, adyacente a la pared anterolateral del ventrículo izquierdo, sin evidencia de compresión. La evaluación del flujograma transmitral, transaórtico y transtricuspídeo no mostró alteraciones sugestivas de compromiso hemodinámico, y la función sistólica del ventrículo izquierdo era normal (Fig. 2).

Debido a los hallazgos ecocardiográficos se le realizó resonancia magnética, mediante la cual se confirmó una imagen quística adyacente al ventrículo izquierdo, de $13.0 \times 10.0 \times 3.7 \mathrm{~cm}$ en sus ejes cefalocaudal, anteroposterior y transversal, respectivamente, sin compresión del ventrículo izquierdo ni reforzamiento tras la administración de gadolinio (Fig. 3). Por las características ya comentadas de la masa quística se diagnosticó quiste pericárdico gigante benigno.

La paciente se encontraba asintomática, por lo que se decidió tratamiento conservador y seguimiento mediante ecocardiogramas seriados.

\section{Discusión}

Las lesiones quísticas del pericardio son lesiones congénitas benignas poco frecuentes, con una incidencia estimada de 1:100,000, y constituyen entre el $4 \%$ y el $7 \%$ de todas las masas mediastinales y el $33 \%$ de los quistes mediastinales. Se diagnostican generalmente entre la tercera y la cuarta décadas de la vida, y tienen una incidencia semejante en hombres y mujeres. Las primeras descripciones datan de mediados del siglo xix en necropsias; los avances en imagenología hicieron posible el diagnostico in vivo y Le Roux reportó tres casos en un grupo de 300,000 pacientes durante una campaña de radiología realizada en Edimburgo en $1958^{3,4}$.

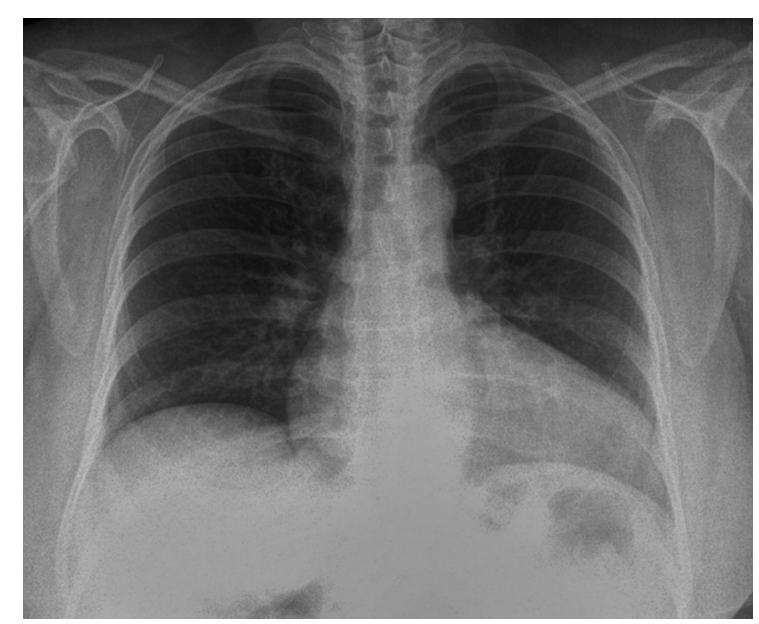

Figura 1. Tele de tórax en la cual se observa cardiomegalia.

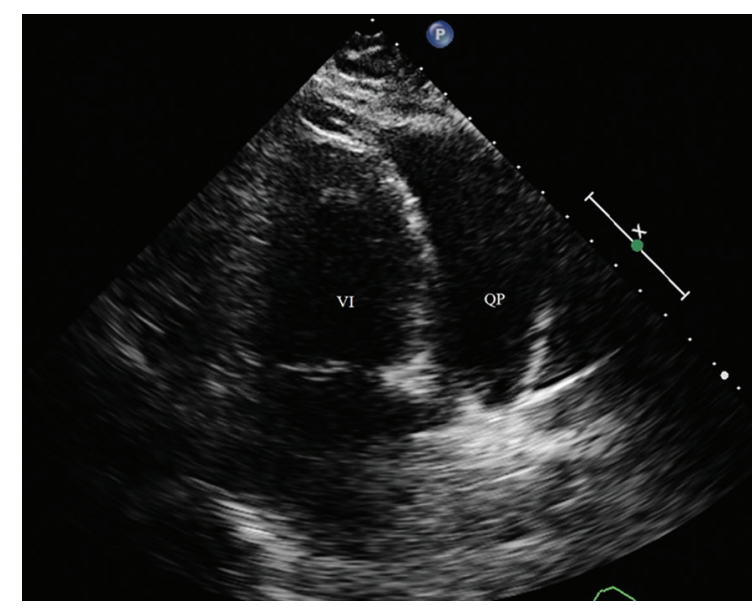

Figura 2. Ecocardiograma transtorácico, aproximación apical de cuatro cámaras, en el que se observa una imagen hipoecoica sugestiva de quiste pericárdico $(Q P)$ adyacente a la pared lateral del ventrículo izquierdo (VI).

Las localizaciones más frecuentes son el ángulo cardiofrénico derecho (51-70\%), el ángulo cardiofrénico izquierdo (28-32\%) y con menos frecuencia otras localizaciones mediastinales no adyacentes al diafragma (8-11\%). En general son no loculados y de diámetro $<3 \mathrm{~cm}^{1,4}$.

Los quistes pericárdicos suelen ser congénitos, de origen mesotelial o celómico, pero existen otras causas, como pericarditis por enfermedades autoinmunitarias, tuberculosis, equinococosis, posterior a cirugía cardiaca, hemodiálisis crónica y quistes malignos (tiroides, linfoma, timoma, teratoma y seminoma) ${ }^{2,4}$.

Los pacientes con quistes pericárdicos son asintomáticos en la mayoría de los casos (50-75\%); sin embargo, 

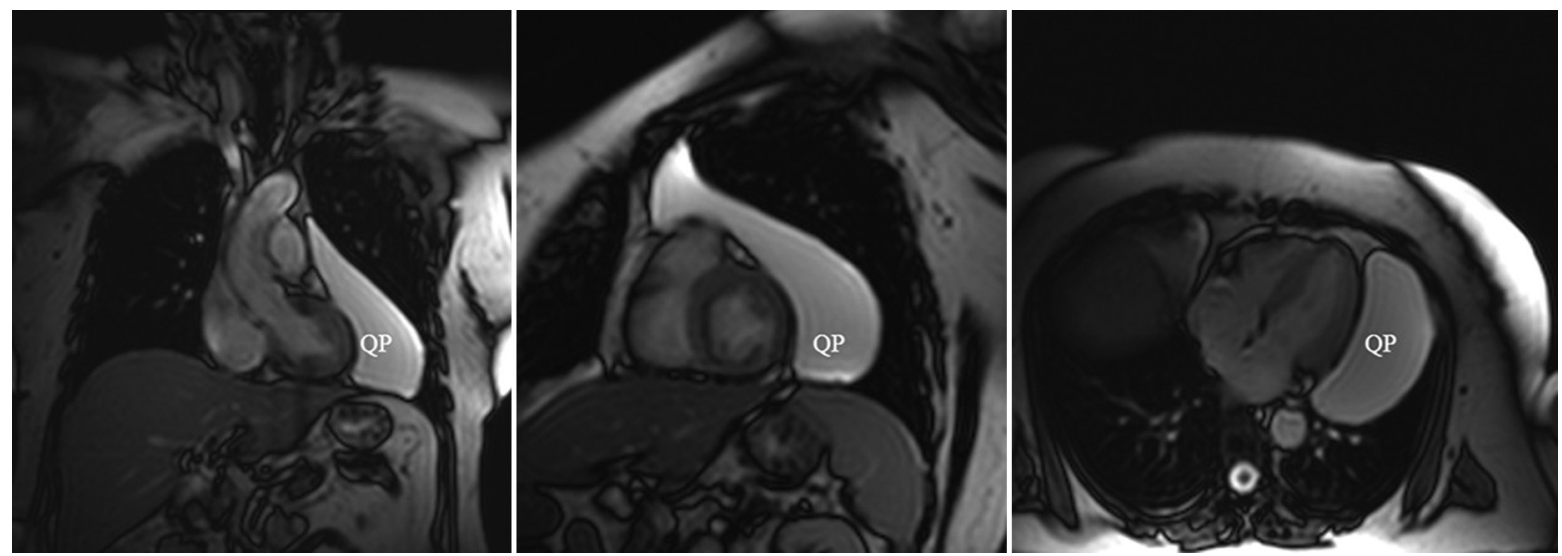

Figura 3. Resonancia magnética en cortes coronal, sagital y axial, en la que se observa un quiste pericárdico (QP) adyacente a la pared lateral del ventrículo izquierdo.

dentro de los reportes de quistes pericárdicos gigantes, con diámetros desde $6.5 \times 9 \mathrm{~cm}$ hasta $21.5 \times 11.4 \times$ $14.2 \mathrm{~cm}$, solo dos pacientes se encontraban asintomáticos (Tabla 1) $)^{1,5-16}$.

La sintomatología puede presentarse cuando el quiste comprime alguna estructura adyacente, como el corazón, la tráquea, los bronquios o el esófago, y los síntomas más comunes son tos crónica, dolor torácico, disnea y opresión retroesternal. Se han descrito también palpitaciones, infecciones respiratorias frecuentes y dolor en el hombro izquierdo como síntomas poco frecuentes ${ }^{2,4,17,18}$.

El diagnóstico suele realizarse por un hallazgo incidental en una radiografía de tórax; otros métodos diagnósticos son el ecocardiograma transtorácico, la tomografía computarizada (TC) y la resonancia magnética (RM). En la TC, los quistes se observan como masas ovaladas, con paredes delgadas y bien definidas, con un coeficiente de atenuación de 30-40 UH, sin realce al administra medio de contraste ${ }^{1}$. En la RM, el líquido contenido en el quiste produce una imagen hiperintensa en las secuencias T2 e hipointensa en las secuencias $\mathrm{T} 1$, sin reforzamiento con el uso de gadolinio 4 .

El ecocardiograma es útil para la evaluación funcional del corazón y para el seguimiento, pero tiene importantes limitaciones en el diagnóstico. Esto se debe a que la localización más frecuente es el lado derecho, donde la imagen obtenida por ecocardiografía es limitada ${ }^{3,4}$.

La American Society of Echocardiography recomienda la realización de TC o RM para confirmar el diagnóstico de un quiste pericárdico cuando se
Tabla 1. Casos reportados de quistes pericárdicos gigantes

\begin{tabular}{|c|c|c|c|}
\hline Referencia & $\begin{array}{l}\text { Dimensiones } \\
(\mathrm{cm})\end{array}$ & Localización & Sintomatología \\
\hline Dernellis, et al. ${ }^{5}$ & $\begin{array}{c}15.6 \times 12.2 \times \\
5.6\end{array}$ & Derecho & Asintomático \\
\hline Nina, et al. ${ }^{6}$ & $13 \times 9.5$ & Derecho & $\begin{array}{l}\text { Disnea, tos, dolor } \\
\text { torácico }\end{array}$ \\
\hline Pereira, et al. ${ }^{7}$ & $14 \times 10 \times 7$ & Bilateral & Dolor torácico \\
\hline Neizel, et al. ${ }^{8}$ & $5 \times 5$ & Posterior & Flutter atrial \\
\hline Matono, et al..$^{9}$ & $12 \times 10$ & Derecho & Asintomático \\
\hline Thanneer, et al. ${ }^{10}$ & $\begin{array}{c}21.5 \times 11.4 \times \\
14.2\end{array}$ & Posterior & Síncope \\
\hline Kumar, et al. ${ }^{11}$ & $10 \times 9.5 \times 9$ & Derecho & $\begin{array}{l}\text { Dolor torácico, } \\
\text { tos, fiebre }\end{array}$ \\
\hline $\begin{array}{l}\text { Kaklikkaya, } \\
\text { et al. }{ }^{12}\end{array}$ & $22 \times 15 \times 7$ & Izquierdo & Dolor torácico \\
\hline Celik, et al..$^{13}$ & $6.5 \times 4.7$ & Izquierdo & Angina de pecho \\
\hline $\begin{array}{l}\text { Forouzandeh, } \\
\text { et al. }{ }^{14}\end{array}$ & $8 \times 5$ & Derecho & Disnea, tos \\
\hline Hamad, et al. ${ }^{15}$ & $11.2 \times 7.4$ & Derecho & $\begin{array}{l}\text { Palpitaciones, } \\
\text { dolor torácico }\end{array}$ \\
\hline Simsek, et al. ${ }^{16}$ & $6.4 \times 9$ & Derecho & $\begin{array}{l}\text { Tos, dolor } \\
\text { torácico }\end{array}$ \\
\hline Hekmat, et al. ${ }^{1}$ & $13 \times 8 \times 5$ & Derecho & Tos, disnea \\
\hline
\end{tabular}

detecta por ecocardiografía, así como para el seguimiento cada 1 o 2 años ${ }^{19}$.

Dentro de las complicaciones que se pueden presentar se encuentran compresión del ventrículo o la aurícula derechos, disfunción diastólica, obstrucción del tracto de 
salida del ventrículo derecho, insuficiencia cardiaca, rotura e infección del quiste pericárdico, hemorragia dentro de la cavidad quística, fibrilación auricular, obstrucción bronquial y neumonía ${ }^{4}$.

El tratamiento depende de la sintomatología. Si el paciente se encuentra asintomático se sugiere únicamente seguimiento, ya sea mediante ecocardiografía, TC o RM. Si el paciente presenta sintomatología, aumento de tamaño del quiste durante el seguimiento o un componente sólido dentro del quiste, el tratamiento de elección es la resección mediante toracotomía, cirugía torácica asistida por video o aspiración percutánea y esclerosis con etanol; sin embargo, con esta técnica puede haber recurrencia hasta en el $33 \%$ de los casos ${ }^{1,4,7,20}$.

\section{Conclusiones}

La mayoría de los quistes pericárdicos son benignos y asintomáticos, excepto los de gran tamaño, los cuales suelen ser sintomáticos.

Generalmente permanecen estables en cuanto a tamaño y sintomatología en los estudios de seguimiento. Se recomienda el ecocardiograma transtorácico como estudio de seguimiento para disminuir la exposición a radiación de la $\mathrm{TC}$, o bien seguimiento con $\mathrm{RM}$. La resección quirúrgica es el único tratamiento definitivo.

\section{Responsabilidades éticas}

Protección de personas y animales. Los autores declaran que para esta investigación no se han realizado experimentos en seres humanos ni en animales.

Confidencialidad de los datos. Los autores declaran que han seguido los protocolos de su centro de trabajo sobre la publicación de datos de pacientes.

Derecho a la privacidad y consentimiento informado. Los autores han obtenido el consentimiento informado del paciente y/o sujeto referidos en el artículo. Este documento obra en poder del autor de correspondencia.

\section{Financiamiento}

El presente documento se financió por los autores.

\section{Conflicto de intereses}

Los autores declaran no tener ningún conflicto de intereses.

\section{Bibliografía}

1. Hekmat M, Ghaderi H, Tatari H, Shabestari AA, Mirjafari SA. Giant pericardial cyst: a case report and review of literature. Iran J Radiol. 2016;13:e21921.

2. Modi S, Chenzbraun A, Fewins H, Binukrishnan S, Ramsdale DR. Giant asymptomatic pericardial cyst. J Cardiovasc Med. 2009;10:646-8.

3. Alkharabsheh S, Gentry III JL, Khayata M, Gupta N, Schoenhagen P, Flamm S, et al. Clinical features, natural history, and management of pericadial cysts. Am J Cardiol. 2019;123:159-63.

4. Kar SK, Ganguly T. Current concepts of diagnosis and management of pericardial cysts. Indian Heart J. 2017;69:364-70.

5. Dernellis J, Theodosiou P, Fois L. An asymptomatic giant pericardial cyst. Int J Cardiol. 2001;2:185-7.

6. Nina VJ, Manzano NC, Mendes VG, Salgado Filho N. Giant pericardial cyst: case report. Rev Bras Cir Cardiovasc. 2007;22:349-51.

7. Pereira A, Tavares NJ, Thomas B. Magnetic resonance imaging of a giant pericardial cyst. Rev Port Cardiol. 2008;27:547-8.

8. Neizel M, Kruger S, Spillner J, Kelm M, Kuhl HP. A giant pericardial cyst as unusual cause for atrial flutter. J Am Coll Cardiol. 2010;55:1160.

9. Matono R, Shoji F, Yano T, Maehara Y. Surgical resection of a giant pericardial cyst showing a rapidly growing feature. Interact Cardiovasc Thorac Surg. 2010;10:1056-8.

10. Thanneer L, Saric M, Perk G, Mason D, Kronzon I. A giant pericardial cyst. J Am Coll Cardiol. 2011:57:1784.

11. Kumar S, Jain P, Sen R, Rattan K, Agarwal R, Garg S. Giant pericardial cyst in a 5-year-old child: a rare anomaly. Ann Pediatr Cardiol. 2011;4:68-70.

12. Kaklikkaya I. A giant pericardial cyst. Cardiovasc J Afr. 2011;22:e1-3.

13. Celik T, Firtina S, Bugan B, Sahin MA, Ors F, lyisoy A. A giant pericardial cyst in an unusual localization. Cardiol J. 2012;19:317-9.

14. Forouzandeh F, Krim SR, Bhatt R, Abboud LN, Ramchandani M, Chang SM. Giant pericardial cyst presenting as pneumonia. Tex Heart Inst J. 2012;39:296-7.

15. Hamad HM, Galrinho A, Abreu J, Valente B, Bakero L, Ferreira RC. Giant pericardial cyst mimicking dextrocardia on chest X-ray. Rev Port Cardiol. 2013;32:49-52.

16. Simsek H, Gunes $Y$, Akil MA, Bisel T. Asymptomatic giant pericardial cyst mimicking dextrocardia on chest X-ray. Herz. 2014;39:1013-5.

17. Patel J, Park C, Michaels J, Rosen S, Kort S. Pericardial cyst: case reports and a literature review. Echocardiography. 2004;21:269-72.

18. Klein AL, Abbara S, Agler DA, Appleton CP, Asher CR, Hoit B, et al. American Society of Echocardiography clinical recommendations for multimodality cardiovascular imaging of patients with pericardial disease. J Am Soc Echocardiogr. 2013;26:965-1012.

19. Maisch B, Seferovci PM, Ristic AD, Erbel R, Rienmuller R, Adler Y, et al. Task Force on the Diagnosis and Management of Pericardial Diseases of the European Society of Cardiology. Guidelines on the diagnosis and management of pericardial diseases executive summary; the Task Force on the Diagnosis and Management of Pericardial Diseases of the European Society of Cardiology. Eur Heart J. 2004;25:587-610.

20. Cangemi V, Volino P, Gualdi G, Polettini E, Frati R, Cangemi B, et al. Pericardial cysts of the mediastinum. J Cardiovasc Surg (Torino). 1999:40:909-13. 